\title{
Azimuthal variations of oxygen abundance profiles in star-forming region of disc galaxies in the EAGLE simulations
}

\author{
Martín Solar $^{1}$, Patricia B. Tissera ${ }^{2 \star}$, Jose A. Hernandez-Jimenez ${ }^{2}$ \\ ${ }^{1}$ Instituto de Física y Astronomía, Facultad de Ciencias, Universidad de Valparaíso, Gran Bretaña 1111, Valparaíso, Chile. \\ ${ }^{2}$ Departamento de Ciencias Físicas, Universidad Andrés Bello, 700 Fernández Concha, Las Condes, Santiago, Chile.
}

Accepted XXX. Received YYY; in original form ZZZ

\begin{abstract}
The exploration of the spatial distribution of chemical abundances in star-forming regions in galactic discs provides clues to understand the complex interplay of physical processes that regulate the star formation activity and the chemical enrichment across a galaxy. We study the azimuthal variations of the normalized oxygen abundance profiles in the highest numerical resolution run of the Evolution and Assembly of GaLaxies and their Environments (EAGLE) Project at $z=0$. We use young stellar populations to trace the abundances of star-forming regions. Oxygen profiles are estimated along different line of sights from a centrally located observer. The mean azimuthal variation in the EAGLE discs are $\sim 0.12 \pm 0.03$ dex $R_{\text {eff }}^{-1}$ for slopes and $\sim 0.12 \pm 0.03$ dex for the zero points, in agreement with previous works. Metallicity gradients measured along random directions correlate with those determine by averaging over the whole discs although with a large dispersion. We find a slight trend for higher azimuthal variations in the disc components of low star-forming and bulge-dominated galaxies. We also investigate the metallicity profiles of stellar populations with higher and lower levels of enrichment than the average metallicity profiles, and we find that high starforming region with high metallicity tend to have slightly shallower metallicity slopes compared with the overall metallicity gradient. The simulated azimuthal variations in the EAGLE discs are in global agreement with observations, although the large variety of metallicity gradients would encourage further exploration of the metal mixing in numerical simulations.
\end{abstract}

Key words: galaxies: evolution, galaxies: abundances, galaxies: ISM

\section{INTRODUCTION}

The standard model for disc formation proposed by Fall \& Efstathiou (1980) is based on the hypothesis of specific angular momentum conservation of the gas as it cools. Numerical simulations have shown that if the condition of angular momentum conservation is globally fulfilled, discs that satisfy the observed scale relations are formed (e.g. Pedrosa \& Tissera 2015; Lagos et al. 2017). In this context, discs form inside-out and, hence, the star formation starts in the central region and moves to the outskirts, contributing to set negative metallicity profiles (e.g. Chiappini et al. 2001; Pilkington et al. 2012b; Tissera et al. 2016). The characteristics of the metallicity profiles provide insight into a vari-

^ E-mail: patricia.tissera@unab.cl ety of physical processes related to galaxy evolution such as nucleosynthesis, stellar winds, star formation (SF) and outflows, among others. These processes can change the oxygen abundance distribution of the interstellar medium (ISM) in a complex way as each of them operates at different time-andspace-scales. Additionally, secular evolution and radial migration, mergers and galaxy-galaxy interactions are dynamical processes that can also modify distribution of chemical abundances in the ISM and the stellar populations (SPs), reshaping the metallicity profiles (Rupke et al. 2010; Amorín et al. 2012; Di Matteo 2016; Grand et al. 2016; Mollá et al. 2016; Tissera et al. 2016; Sillero et al. 2017; Ma et al. 2017; Tissera et al. 2019).

In the Local Universe, HII regions in spiral galaxies are known to have negative abundance gradients, on average, (e.g. Searle 1971; Martin \& Roy 1992; Zaritsky et al. 
1994; Kennicutt et al. 2003; Rosales-Ortega et al. 2011) so that the inner regions are more metal-rich than the outskirts. However, departures from a single metallicity gradients (Sánchez-Menguiano et al. 2017) and the existence of inverted metallicity gradients associated to interacting galaxies have been also reported (e.g. Rupke et al. 2010; Rosa et al. 2014). First observations of nearby spiral galaxies showed no clear indiction of important azimuthal variations in the metallicity distributions (e.g. Martin \& Belley 1996; Cedrés \& Cepa 2002; Cedrés et al. 2012; Kennicutt \& Garnett 1996; Li et al. 2013; Zinchenko et al. 2016). Only recently, with the help of Integral Field Spectroscopic (IFS) surveys, it has been possible to analyse the metallicity distributions across the discs in more detail and with a larger statistical significance. Sánchez-Menguiano et al. (2017) analysed 63 face-on spiral galaxies selected from CALIFA data, finding differences in the chemical abundances of the arms and interarms regions, with modal values of $-0.013 \operatorname{dex} R_{\text {eff }}^{-1}$ and $-0.015 \operatorname{dex} R_{\text {eff }}^{-1}$ for flocculent and grand design spirals, respectively. Detailed observations of individual galaxies also provide evidences of azimuthal variations (Li et al. 2013; Sánchez-Menguiano et al. 2016; Vogt et al. 2017; Ho et al. 2017). For example, Ho et al. (2018) analysed the oxygen abundance of HII regions in NGC 2997, reporting 0.06 dex azimuthal variations in the oxygen abundance, with higher enrichment in the arm regions. Although the reported azimuthal variations are small, they provide information on the regulation of the SF activity and metal mixing process across the discs.

From a theoretical point of view, Grand et al. (2016) used high resolution hydrodynamical simulations to follow the evolution of radial flows associated to the spiral arms. These authors showed how they could produce an overdensity of high-metallicity stars in the trailing side of the arms and an overdensity of metal-poor stars on the leading side. Di Matteo (2016) reported that radial migration induced by a bar can produce azimuthal variation of the old stellar populations using pure N-body simulations. Khoperskov et al. (2018) analysed the formation of azimuthal metallicity variations using high resolution N-body simulations of discs with no initial metallicity gradients. They found that the different responses to a spiral perturbation of the kinematically hot and cold stellar populations produce variations in the metallicity distributions. Using analytical 2D chemical models, Spitoni et al. (2018) study and quantify the effects of spiral arm density fluctuations on the azimuthal metallicity variations. These authors report an azimuthal variation of the metallicity gradients of the order of $\sim 0.1$ dex.

Numerical simulations that include chemical models, such as the simulations from the Evolution and Assembly of GaLaxies and their Environment (EAGLE) Project (Crain et al. 2015; Schaye et al. 2015), are powerful tools to contrain the subgrid models for the evolution of baryons. Chemical patterns give additional information on the processes that regulate the star formation activity and redistribute the angular momentum and mass in galaxies as well as on the impact of inflows and outflows. Because they are usually not used to fix the free parameters of the algorithms of the subgrid physics, their analysis provide constraints to them. The chemical elements are often distributed within the nearby regions of the stellar sources (Mosconi et al. 2001). Unless other mechanisms are included, there will no further exchange of material within the minimum resolved volumes. Metal diffusion is expected to contribute to mitigate this issue at the expense of introducing an extra free parameter, the metal diffusion coefficient (Greif et al. 2009; Pilkington et al. 2012a). The characteristics of the metal distribution and the gradients will be affected by the efficiency of the mixing and/or diffusion of chemical elements. This is an important issue considering that the cooling rates depend strongly on the chemical abundances of the ISM for example. The more detailed observational data that are being gathered by IFS surveys open the possibility to perform more detail comparisons with models and hence, we expect the analysis presented in this paper to serve as a benchmark for future improvements.

In this work, we analyse the azimuthal variation of oxygen abundances of the discs identified from a set of simulated galaxies extracted from the high-resolution $(25 \mathrm{Mpc})^{3}$ volume of the EAGLE Project. The EAGLE simulations have proven to reproduce global properties of galaxies such as the mass-metallicity relation (De Rossi et al. 2017), the fundamental relations of early type galaxies (Lagos et al. 2018; Trayford et al. 2019; Rosito et al. 2018), and the locallyresolved scale relation between star formation and metallicity (Trayford \& Schaye 2019), among others. In particular, these simulations provide a large sample of galaxies with different formation histories, which opens the possibility to assess the existence of azimuthal metallicity variations. This work is based on the galaxy catalogue built by Tissera et al. (2019) from which disc galaxies were selected for the higher resolution run of $25 \mathrm{Mpc}$ cubic-side volume. In this paper, we analyse the azimuthal variations of the oxygen abundances in star-forming regions in the disc components at $z=0$.

This paper is organised as follows. Section 2 describes the simulations and methods implemented to select the analysed stellar populations. Section 3 presents the analysis of the azimuthal variations of the chemical abundance distributions. In Section 4 we summarises our main results. The Appendix provide information on the impact of the numerical dispersion in the metallicity distribution.

\section{SIMULATIONS}

The EAGLE Project ${ }^{1}$ comprises cosmological hydrodynamical simulations consistent with a $\Lambda$-CDM universe, performed assuming $\Omega_{\Lambda}=0.693, \Omega_{\mathrm{m}}=0.307, \Omega_{\mathrm{b}}=0.04825$, $h=0.6777\left(H_{0}=100 \mathrm{~h} \mathrm{~km} \mathrm{~s} \mathrm{Mpc}^{-1}\right), \sigma_{8}=0.8288$, $n_{\mathrm{s}}=0.9611$, and $Y=0.248$ (Planck Collaboration et al. 2014). The simulations were performed with an enhanced version of the GADGET-2 code (Springel 2005) that includes a modified hydrodynamics solver and time stepping by the ANARCHY model (Schaller et al. 2015).

The code tracks the chemical enrichment of eleven chemical elements produced by mass loss by intermediatemass asymptotic giant branch, Type Ia and II supernovae as well as mass winds by massive stars (Wiersma et al. 2009b). A Chabrier (2003) initial mass function is adopted.

1 We use the publicly available database by McAlpine et al. (2016). 
It also includes radiative cooling and a photo-heating models as described in Wiersma et al. (2009a). The energy feedback model from stellar sources is implemented stochastically (Dalla Vecchia \& Schaye 2012) and was calibrated to reproduce the stellar mass function and galaxy sizes at $z=0$. More details of the simulations and the implemented subgrid physics can be found in Crain et al. (2015).

For this study, we use the re-calibrated simulation (RefL025N0752) representing a volume of $25 \mathrm{Mpc}$ comoving box side, resolved by $752^{3}$ initial particles. The mass resolution is $2.26 \times 10^{5} \mathrm{M}_{\odot}$ and $1.21 \times 10^{6} \mathrm{M}_{\odot}$ for the initial gas and dark matter particles, respectively. The maximum proper gravitational softening is $0.35 \mathrm{pkpc}$.

The EAGLE project also provides smoothed chemical abundances, which are estimated by applying a kernel function used to calculate smoothed values by using the information of the nearby regions. The smoothed abundances are used to estimate the cooling rates in the EAGLE simulations. Hence, in order to check their impact and for sake of consistency, we repeated the whole analysis of the metallicity distributions by using the smoothed abundances in order to assess if the results depend on this mixing. We detect no significant change in the azimuthal variations neither in the trends reported by using the non-smoothed abundances. Selected trends obtained using the smoothed abundances are included in the Appendix A as examples.

\subsection{Galaxy sample}

The simulated galaxy sample is selected from the catalogue of Tissera et al. (2019), where a dynamical criteria based on the angular momentum context and binding energy was applied to identify the disc components (Tissera et al. 2012). The angular momentum content of each particle is quantified by $\epsilon=J_{\mathrm{Z}} / J_{\mathrm{Z}, \max }(E)$, where $J_{\mathrm{Z}}$ is the angular momentum and $J_{\mathrm{Z}, \max }(E)$ is the maximum $J_{\mathrm{z}}$ over all particles at a given binding energy $E$. Only stars with $\epsilon>0.5$ are taken into count to define the stellar disc component. Stars particles that are not rotational supported are considered to belong to the spheroidal component and are not analysed in this work (see Rosito et al. 2018).

For the purpose of our analysis, we define two sets of SPs: young stars which are selected to have ages smaller than 2 Gyr and super-young stars, chosen to have ages younger than 0.5 Gyr. For each simulated galaxies, the star formation rate (SFR), the specific star formation rate (sSFR), the halfmass radius $\left(R_{\text {eff }}\right)$, defined as the one that encloses half the mass of the young (super-young) stellar discs are estimated. Galaxy morphology is defined by the ratio between the total stellar mass of the discs and the total stellar mass of a galaxy $(\mathrm{D} / \mathrm{T})$. The abundance ratio $12+\log (\mathrm{O} / \mathrm{H})$ will be used to quantify the level of enrichment of the simulated SPs (hereafter, we will also use the term 'metallicity' to refer to the oxygen abundances).

We follow previous works that showed that young SPs can be taken as tracers of the chemical abundances of the star-forming regions in the ISM (Gibson et al. 2013; Tissera et al. 2016). We adopt the same criteria considering the two mentioned age thresholds which will be used for different aspects of the analysis. Only those discs with more than 1000 young (super-young) stars will be analysed. This condition is adopted because the estimation of the metallic- ity gradients in different azimuthal direction requires to be able to numerically resolve all of them with a suitable number of star particles. The final samples comprise 106 and 42 discs (sampled by young stars and super-young stars, respectively). The selected galaxies have stellar masses in the range $\left[10^{9}, 10^{10.8}\right] \mathrm{M}_{\odot}$ and SFR in range $[0.1,6] \mathrm{M}_{\odot} y r^{-1}$.

In order to illustrate the distribution of both metallicity and mass of the SPs studied, Fig. 1 shows the face-on projections of metallicity (left-panels), the face-on (middlepanels) and edge-on (right-panels) projections of the stellar mass density for the super-young, young and old (with ages $>2$ Gyr) SPs. To create these maps, we used grids of $50 \times 50$ pixel on images with a physical size of $50 \times 50 \mathrm{kpc}$. For the face-on metallicity projection, each pixel was assigned the median value along z-axis, while for the face-on and edgeon stellar mass density projections were assigned the sum of the stellar mass along z- and y-axes, respectively. As expected both super young and young SPs are good tracers of the spiral arms as denoted by the iso-density contours on the face-on images and are distributed in thin disc as shown in edge-on projections, while the old SP traces the disc and is distributed in a thick disc (see face-on and edgeon projections, respectively). The metallicity distributions of super-young and young SPs have larger contribution of high metallicity SPs $(12+\log (\mathrm{O} / \mathrm{H})>8.5$, red points $)$, while the old SPs seem to have more homogeneous values around of $12+\log (\mathrm{O} / \mathrm{H}) \sim 8.5$ (white points). The different spatial and numerical distributions of metallicity among the SPs will be quantified in more detail in the following sections.

\section{AZIMUTHAL VARIATIONS OF THE OXYGEN PROFILES}

In this section, we quantify the azimuthal variations of the oxygen abundance gradients by estimating the metallicity profiles along different directions on the disc plane from an observed located at the galactic centre. Each galaxy is rotated so that the total angular momentum is located along the z-axis. The stellar discs are projected along the z-axis. For simplicity, the azimuthal variations are obtained by tessellating in six equal sub-regions the projected stellar mass distributions, along the main axis of rotation. Each of the resulting regions covers $60^{\circ}$ of the total disc $\left(360^{\circ}\right)$. The radial metallicity profiles are obtained by estimating the median oxygen abundance values in radial intervals, each one enclosing the same number of star particles ${ }^{2}$. We only considered sub-regions with at least 200 young SPs, so each of them had enough star particles to estimate the metallicity profiles. In order to have enough number of SPs in each subregions, in this section, we work with the young SPs (ages $<2$ Gyr).

Linear regression fittings to the metallicity profiles are constructed by applying the least trimmed squares (LTS) robust method that considers errors in the y-variable, leaving out possible outliers (Rousseeuw \& Van Driessen 2006; Cappellari et al. 2013). The errors correspond to three times

2 A total of 20 radial intervals are defined in each disc resolved with $\mathrm{N}$ particles, within $[0.5,1.5] R_{\text {eff }}$. The widths are determined by the distributions of $n$ particles, where $n=N / 20$. 

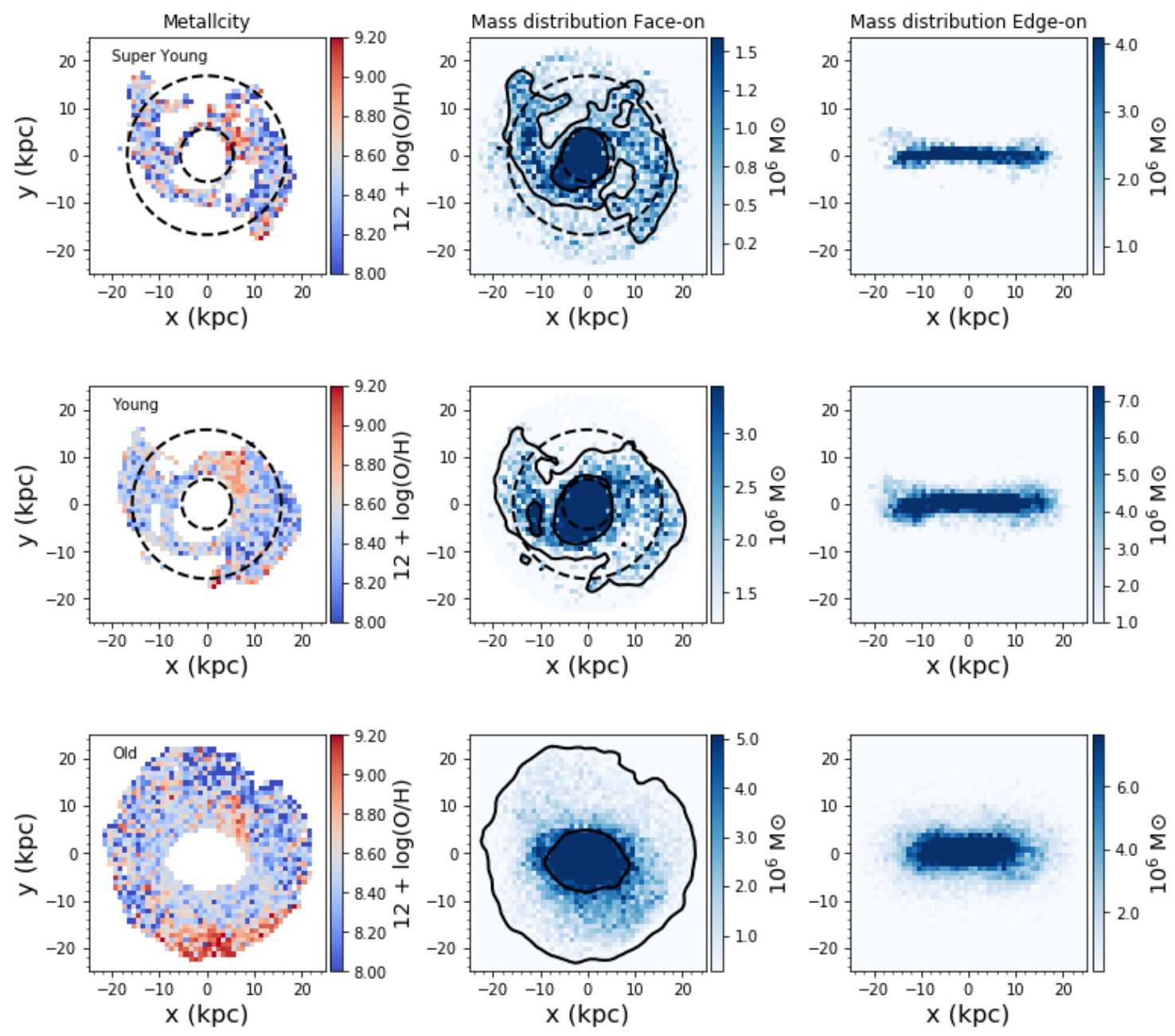

Figure 1. Metallicity and stellar mass distributions of a galaxy with well-defined spiral arms from our selected sample. Lelf panels: faceon projections of the oxygen abundance $(12+\log (\mathrm{O} / \mathrm{H}))$ for the super-young (top), young (middle) and old (bottom) populations. Middle panels: face-on projections of stellar mass density of star particles for super-young (top), young (middle) and old (bottom) populations. The inner and outer black circles represent 0.5 and $1.5 R_{\text {eff }}$, respectively. The black contours represent mass iso-densities chosen to highlight the arm and disc structures for young (top and middle) and old (bottom) populations, respectively. The face-on metallicity projections were masked inside of the mass iso-density contours shown in the middle panels. Right panels: the same as middle panels but for edge-on projections of stellar mass density.

the bootstrap errors estimated for each radial interval ${ }^{3}$. A linear regression of the form: $y=b\left(x-x_{0}\right)+a$ is used in all cases. It should be noted that the metallicity profiles are normalized by $R_{\text {eff }}$, before applying the LTS fits over the radial range $[0.5,1.5] R_{\text {eff }}$.

After this procedure, six slopes $(\nabla)$ and six zero points $(Z P)$ for each galaxy are obtained. We estimated the ZPs at the interception with $x=0$. The median azimuthal slopes $\left(\nabla_{\mathrm{M}}\right)$ and zero points $\left(Z P_{\mathrm{M}}\right)$ were estimated from the linear regressions calculated for the six sub-regions. Their respective standard deviations are obtained by applying a bootstrap technique $\left(\sigma_{\nabla}\right.$ and $\left.\sigma_{\mathrm{ZP}}\right)$. Additionally, the overall metallicity gradients were also calculated by using concen-

\footnotetext{
3 Bootstrap errors are used instead of the standard dispersion in each interval because the analysis of the metallicities distributions shows that the dispersions have a systematic skewness with a lower metallicity tail at all radius
}

tric radial averages $\left(\nabla_{\mathrm{T}}\right.$ and $\left.Z P_{\mathrm{T}}\right)$. In summary, for each simulated galaxy, we have four parameters: $\nabla_{\mathrm{T}}, \sigma_{\nabla}, Z P_{\mathrm{T}}$, and $\sigma_{\mathrm{ZP}}$.

Figure 2 shows the metallicity profiles obtained for each of the six sub-regions of the a typical simulated disc (black, dashed lines) compared to the overall metallicity profile for the whole disc (red, solid lines) as an example. As it can appreciate from Fig. 2, there are clear azimuthal variations of the slopes and zero points of the metallicity profiles of each sub-region.

Figure 3 displays the metallicity gradients and zero points estimated along one of the defined azimuthal subsamples selected at random, $\nabla_{\text {Random }}$ and $Z P_{\text {Random }}$, as a function of $\nabla_{\mathrm{T}}$ and $Z P_{\mathrm{T}}$. The gradient and zero point of the metallicity profiles taken at random from our set of six directions correlates with those obtained from the radial averages over the whole discs, indicating that even if there is azimuthal variations, the information store in the overall profiles can be recovered by using measures along a certain direction. 


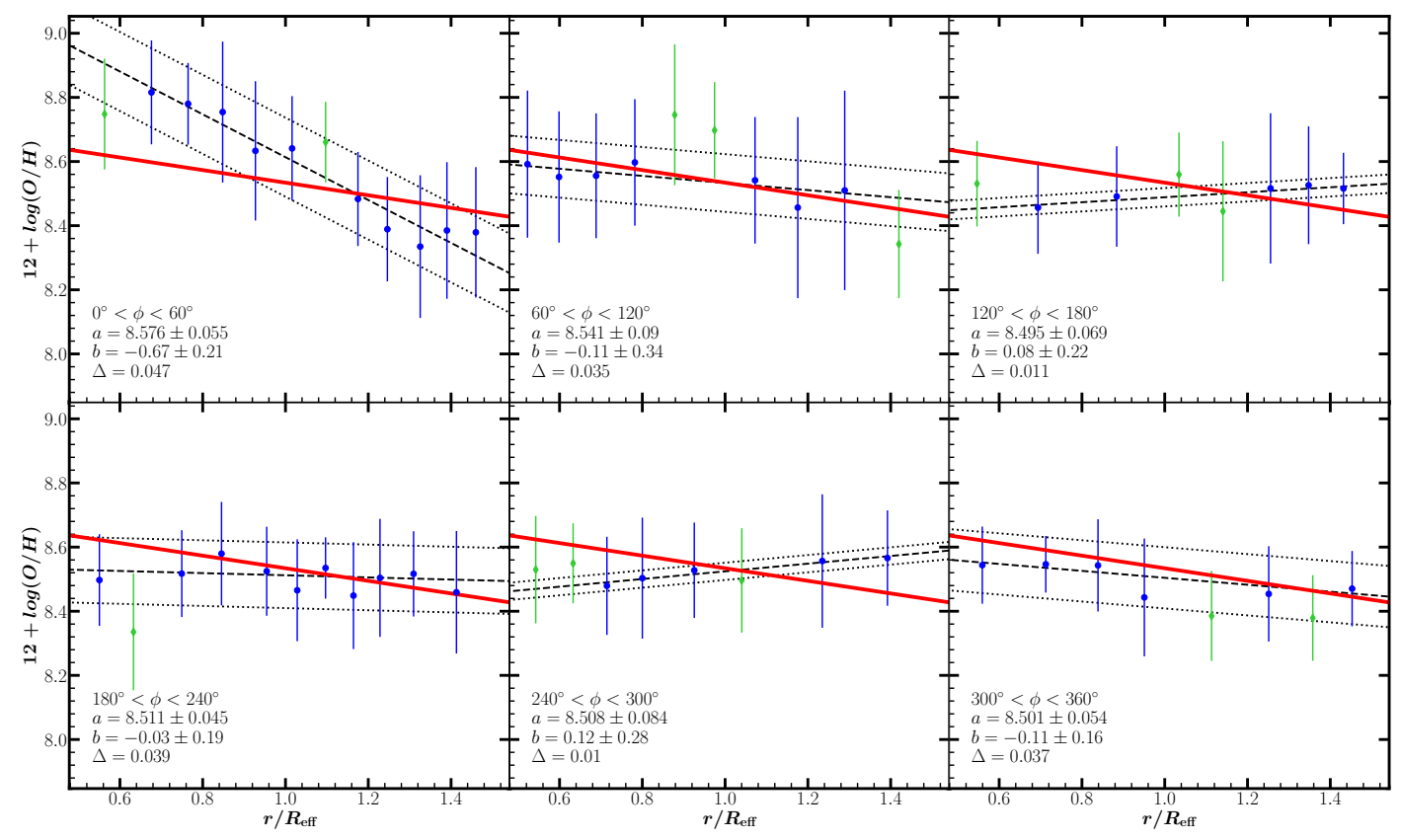

Figure 2. Oxygen abundance profiles of the SF regions located in each of the six sub-regions defined to assess the azimuthal variations for typical galaxy (black dashed lines). The error bars denote three times the bootstrap errors. For comparison, the global averaged profile is also shown (red thick line). The standard metallicity deviations in each radial are included (only blue points are considered for the LTS fist while green symbols are classified as outliers (Rousseeuw \& Van Driessen 2006; Cappellari et al. 2013)). The black dotted lines are the linear fits of LTS $\pm 2.6 \triangle$ (standard deviation).

However, as it can be seen from this figure, both relations show large dispersion. The Spearman coefficient of correlations are $r \sim 0.46$ and $r \sim 0.43$ for the slopes and zero points, respectively. This suggests that the azimuthal variations are significant.

The relation between the azimuthal dispersions of the slopes $\sigma_{\nabla}$ and those of the zero points $\sigma_{\mathrm{ZP}}$ is depicted in the Fig. 4. As can be seen there is a trend for discs with larger $\sigma_{\nabla}$ to also have larger azimuthal variations of the zero point. The error bars are estimated by using a bootstrap technique. The medians values are $\sigma_{\nabla} \sim 0.12 \pm 0.03 \operatorname{dex} R_{\text {eff }}^{-1}$ and $\sigma_{\mathrm{ZP}} \sim 0.12 \pm 0.03$ dex, respectively.

To explore the origin of the azimuthal variation of the metallicity profiles, Fig. 5 shows $\sigma_{\nabla}$ and $\sigma_{\mathrm{ZP}}$ as a function of $D / T$ (upper panels), $R_{\text {eff }}$ (middle panels), and logSFR (lower panels) coloured by $\nabla_{\mathrm{T}}$ (left panels) and $Z P_{\mathrm{T}}$ (right panels). The median values and the 25 and 75 percentiles (error bars) are also included. We find weak trends of the azimuthal dispersions in the gradients as a function of $D / T$ and SFR. These indicate slightly higher azimuthal variations of the metallicity distributions in discs of more bulge-dominated systems and low SFR galaxies. We would like to point out that the larger azimuthal variation of the metallicity distributions detected for galaxies with low star- formation activity could be also affected by numerical resolution, and hence, these trend should be confirmed by using higher resolution simulations. No clear trend is found for the azimuthal varia- tions of the zero points of the metallicity profiles. There are no clear trends of both dispersions with $R_{\text {eff }}$.

The fact that disc-dominated galaxies show less azimuthal dispersion in the metallicity gradients could be associated to their more quiescent evolution history where the ISM got progressively enriched and the stellar distributions were not strongly disturbed. On the other hand, bulge-dominated galaxies had a larger probability to have experienced mergers or interactions in the past as shown by Tissera et al. (2019). We speculate that these mechanisms could have disturbed the stellar distributions and hence, increase the azimuthal dispersion, forming a bar for example. The impact would depend on the merger/internaction parameters and how close in time from $z \approx 0$ these event had taken place. A more detailed analysis of possible mixing processes would require better space and temporal resolutions. Well-defined disc galaxies $(D / T \geq 0.5)$ in the EAGLE simulation have azimuthal variations in the metallicity gradients of $\sim 0.1$ dex $R_{\text {eff }}$. This value is in agreement with the results reported by (Spitoni et al. 2018) for spirals using chemodynamical model are analysed.

\section{OXYGEN PROFILES IN ARM AND INTERARM REGIONS}

Another approach for studying the azimuthal metallicity variations is the analysis the residual metallicity distribu- 

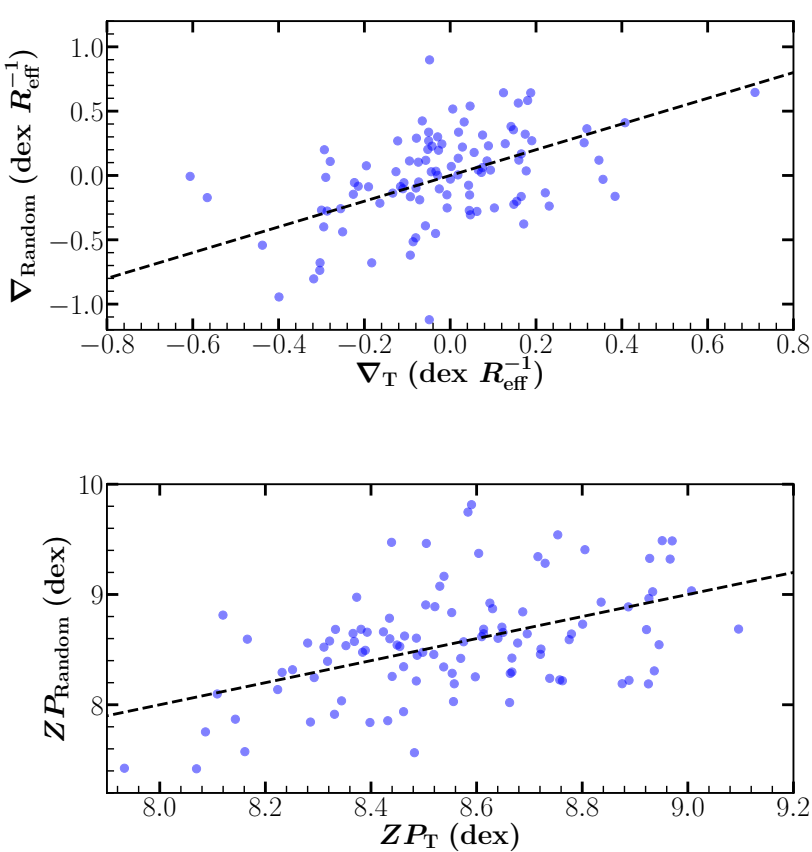

Figure 3. Metallicity gradient $\nabla_{\text {Random }}$ estimated along an azimuthal sub-sample selected at random from the six defined ones as a function of the global metallicity slopes $\nabla_{\mathrm{T}}$ (upper panel) and the corresponding zero points $Z P_{\text {Random }}$ as a function of the global ones $Z P_{\mathrm{T}}$ (lower panel).



Figure 4. Median values of the standard deviations of the azimuthal zero points $\sigma_{\mathrm{ZP}}$ as a function of those of $\sigma_{\nabla}$ (black squares). The black error bars represent the 25 and 75 percentile. The blue small dots and blue error bars represent the data for individual simulated galaxies and the corresponding errors obtained by applying a bootstrap sampling technique over the six azimuthal subregions.


$\nabla_{\mathrm{T}}\left(\operatorname{dex} R^{-1}\right)$



$Z P_{\mathrm{T}}(\mathrm{dex})$



$\nabla_{\mathrm{T}}\left(\operatorname{dex} R_{\mathrm{eff}}^{-1}\right)$


Figure 5. $\sigma_{\nabla}$ (left panels) and $\sigma_{\mathrm{ZP}}$ (right panels) as a function of $D / T$ (upper panel), $R_{\text {eff }}$ (middle panel), and SFR (lower panel). The colour bars show $\nabla_{\mathrm{T}}$ (left panels) and $Z P_{\mathrm{T}}$ (left panels). Median values are shown (black squares) with error bars representing the 25 and 75 percentiles.

tions. With the super-young SPs, we estimate the overall metallicity profiles and obtained $\nabla_{\mathrm{T}}$ and $Z P_{\mathrm{T}}$, following the same procedure explained in the previous section. Then, the SPs that have an excess or deficit of oxygen abundances with respect to the overall metallicity profile are identified by defining the residues $(\delta)$,

$\delta\left(r_{i}\right)=Z_{\text {real }}\left(r_{i}\right)-Z_{\mathrm{fit}}\left(r_{i}\right)$

where $Z_{\text {real }}\left(r_{i}\right)$ is the metallicity of a given super-young SPs located at a galacto-centric distance $r_{i}$ and $Z_{\mathrm{fit}}\left(r_{i}\right)$ is the metallicity it should have at that $r_{i}$, according to the overall linear fit. We note this procedure is applied to all selected stars in order to build up the metallicity residual maps. We choose to use the fitting relations as reference values because this is the usual way of quantifying the metallicity distributions at any redshift (Carton et al. 2018) and they allow us to have a reference value to define the residuals for each individual SP. Hence, each super-young SP is classified as having a metallicity excess $\left(\delta_{+}\right)$or deficit $\left(\delta_{-}\right)$with respect to the overall level of enrichment. The radial metallicity profiles of the super-young SPs with $\delta_{+}$and $\delta_{-}$are estimated following the same procedure explained in the previous sec- 
tion $\left(\nabla_{\delta_{+}}\right.$and $Z P_{\delta_{+}}$and $\nabla_{\delta_{-}}$and $Z P_{\delta_{-}}$, respectively). In Fig. 6 , we show the gradients and zero points determined by the regions with excess versus those with deficitwith respect to the overall metallicity profiles. As can be appreciated there is a slight trend for the regions with excess metallicity to show weaker gradients. From this figure, it can be appreciated a systematic shift for the zero point values of the excess and deficitprofiles, with respect to the $Z P_{\mathrm{T}}$ of the global metallicity profiles, respectively. The difference between the zero points of the regions with excess and deficit metallicities with respect to the median values is smaller for the galaxies with higher metallicity. This suggests a higher mixing of chemical elements or/and a prolonged SF history.

Figure. 7 shows the relation of $\nabla_{\delta_{-}}$versus $\nabla_{\delta_{+}}$together with the metallicity gradients reported by SánchezMenguiano et al. (2017) are displayed for the sake of comparison. However, it is important to stress that the latter were obtained by applying a different method. SánchezMenguiano et al. (2017) estimated the metallicity gradients for HII regions located in the arm and interarm regions. Nevertheless, both approaches intent to highlight the difference in the metallicity distribution of the SPs in the discs. It is clear that the simulated parameters show large dispersion which, at least in part, could be due to inefficient mixing of the chemical elements or more clumpy star-formation activity.

In order to complete the analysis, we explore if the smoothed abundances could reduce the scatter by repeating this analysis with the smoothed element abundances as shown in the Appendix. As can be seen from Fig. A2, using the smoothed abundances does not reduce the scatter in the metallicity gradients or reduce the gap between the level of enrichment between the SPs classified as having metallicity excess or deficit.

\section{CONCLUSIONS}

We study the azimuthal variation of the oxygen abundances of young and super-young SPs which can be used as tracers of the regions of active SF in the discs of galaxies selected from the higher resolution run of the EAGLE project, Ref-L025N0752. The azimuthal variations of the metallicities can store information on the evolution of discs and currently, IFS observations provide detail information of the metallicity distributions. Additionally, they provide another route to confront the subgrid physics with observations. Our analysed sample is made of 106 (42) galaxies resolved with at least 1000 young (super-young) star particles. As a consequence, our sample represents star-forming galaxies with $\mathrm{SFR}>0.1 \mathrm{M} \odot \mathrm{yr}^{-1}$.

Our main results can be summarized as follows:

- Although we find a correlation between the metallicity gradients measured along a random direction and those estimated by using global averages over the discs, the scatter is large enough to suggest azimuthal variations are significant. The azimuthal dispersion of the slopes and zero points of young stars in the EAGLE discs are found to be around $0.12 \pm 0.03$ dex $R_{\text {eff }}^{-1}$ and $0.12 \pm 0.03$ dex, respectively.

- A weak trend to have larger metallicity azimuthal dispersions are detected for galaxies with lower star-formation


Figure 6. Upper panel: $\nabla_{\delta_{+}}$(green dots) and $\nabla_{\delta_{-}}$(red squares) as a function of $\nabla_{\mathrm{T}}$ estimated for the whole distribution of superyoung stars. Lower panel: $Z P_{+}$(green dots) and $Z P_{-}$(red squares) as a function of $Z P_{\mathrm{T}}$. The black dashed line corresponds to $1: 1$ relation in both plots.

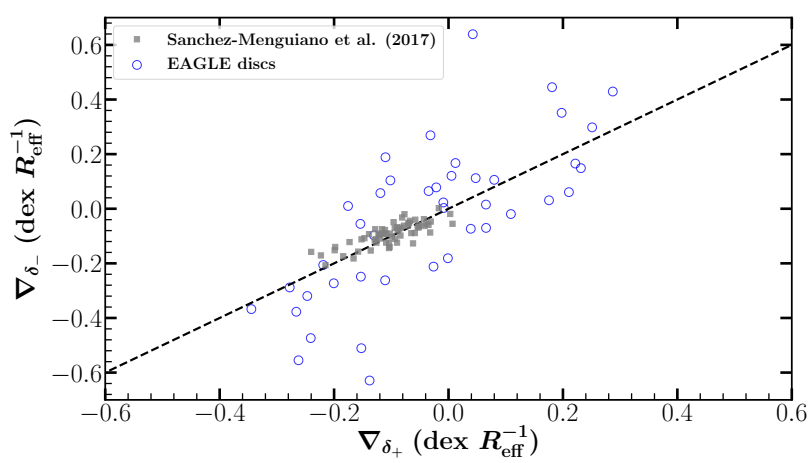

Figure $7 . \nabla_{\delta_{+}}$as a function of $\nabla_{\delta_{-}}$defined by super-young stars (blue circles). The black dashed line corresponds to the 1:1 relation. Observations form Sánchez-Menguiano et al. (2017) have been included only for reference (gray points) since they estimated the gradients by selected HII regions located in the arm and interarm regions. 
activity and $D / T<0.2$. The larger variation for bulgedominated galaxies and low star-formation activity is in agreement with the results obtained by Tissera et al. (2019), where the gas-phase oxygen profiles in the star formation regions were analysed for galaxies of different morphologies in the EAGLE simulations. Previous works reported bulge-dominated systems to have larger frequency of mergers. These events could have destroyed part of the discs or disturbed them by forming bar or spiral structures, which could have contributed to mix up the stellar populations. It has also to be considered that these systems have lower star-formation activity and, even though we required a minimum number of stars to make the estimations, the dispersion could be more affected by numerical resolution than those discs with larger star-formation rates. However, currently, it is still difficult to have a large dataset of galaxies with a vast variety of morphologies and at the same time, with high numerical resolution. Well-defined discs with $D / T>0.4$ show azimuthal dispersions of 0.1 dex $R_{\text {eff }}^{-1}$ in good agreement with the predictions by Spitoni et al. (2018). We find no clear trends with $R_{\text {eff }}$.

- The metallicity slopes estimated by using the superyoung SPs with metallicity excess are slightly shallower than the overall estimations. However, the larger variation of these gradients in the simulations with respect to the current observations (e.g. Sánchez-Menguiano et al. 2016) suggests the relevance of exploring in more detail the flow of metals at sub-galactic scales.

\section{ACKNOWLEDGMENTS}

The authors thank to A. Benitez-Llambay and J.Schaye for useful comments. JAHJ thanks to CONICYT, Programa de Astronomía, Fondo ALMA-CONICYT 2017, Código de proyecto 31170038. PBT acknowledges partial funding by Fondecyt Regular 2015 - 1150334 and Internal Project Unab 2019. This project has received funding from the European Union Horizon 2020 Research and Innovation Programme under the Marie Sklodowska-Curie grant agreement No 734374. This worked used the RAGNAR cluster funded by Fondecyt 1150334 and Universidad Andres Bello. This work used the DiRAC Data Centric system at Durham University, operated by the Institute for Computational Cosmology on behalf of the STFC DiRAC HPC Facility (www.dirac.ac.uk). This equipment was funded by BIS National E-infrastructure capital grant ST/K00042X/1, STFC capital grants ST/H008519/1 and ST/K00087X/1, STFC DiRAC Operations grant ST/K003267/1 and Durham University. DiRAC is part of the National E-Infrastructure. We acknowledge PRACE for awarding us access to the Curie machine based in France at TGCC, CEA, Bruyeresle-Chatel.

\section{REFERENCES}

Amorín R., Vílchez J. M., Hägele G. F., Firpo V., Pérez-Montero E., Papaderos P., 2012, ApJ, 754, L22

Cappellari M., et al., 2013, MNRAS, 432, 1709

Carton D., et al., 2018, MNRAS,

Cedrés B., Cepa J., 2002, A\&A, 391, 809
Cedrés B., Cepa J., Bongiovanni Á., Castañeda H., SánchezPortal M., Tomita A., 2012, A\&A, 545, A43

Chabrier G., 2003, ApJ, 586, L133

Chiappini C., Matteucci F., Romano D., 2001, ApJ, 554, 1044

Crain R. A., et al., 2015, MNRAS, 450, 1937

Dalla Vecchia C., Schaye J., 2012, MNRAS, 426, 140

De Rossi M. E., Bower R. G., Font A. S., Schaye J., Theuns T., 2017, MNRAS, 472, 3354

Di Matteo P., 2016, Publications of the Astronomical Society of Australia, 33, e027

Fall S. M., Efstathiou G., 1980, MNRAS, 193, 189

Gibson B. K., Pilkington K., Brook C. B., Stinson G. S., Bailin J., 2013, A\&A, 554, A47

Grand R. J. J., et al., 2016, MNRAS, 460, L94

Greif T. H., Glover S. C. O., Bromm V., Klessen R. S., 2009, MNRAS, 392, 1381

Ho I. T., et al., 2017, ApJ, 846, 39

Ho I. T., et al., 2018, A\&A, 618, A64

Kennicutt Robert C. J., Garnett D. R., 1996, ApJ, 456, 504

Kennicutt Robert C. J., Bresolin F., Garnett D. R., 2003, ApJ, 591,801

Khoperskov S., Di Matteo P., Haywood M., Combes F., 2018, A\&A, 611, L2

Lagos C. d. P., Theuns T., Stevens A. R. H., Cortese L., Padilla N. D., Davis T. A., Contreras S., Croton D., 2017, MNRAS, 464,3850

Lagos C. d. P., et al., 2018, MNRAS, 473, 4956

Li Y., Bresolin F., Kennicutt Robert C. J., 2013, ApJ, 766, 17

Ma X., Hopkins P. F., Wetzel A. R., Kirby E. N., Anglés-Alcázar D., Faucher-Giguère C.-A., Kereš D., Quataert E., 2017, MNRAS, 467, 2430

Martin P., Belley J., 1996, ApJ, 468, 598

Martin P., Roy J.-R., 1992, ApJ, 397, 463

McAlpine S., et al., 2016, Astronomy and Computing, 15, 72

Mollá M., Díaz Á. I., Gibson B. K., Cavichia O., López-Sánchez Á.-R., 2016, MNRAS, 462, 1329

Mosconi M. B., Tissera P. B., Lambas D. G., Cora S. A., 2001, MNRAS, 325, 34

Pedrosa S. E., Tissera P. B., 2015, A\&A, 584, A43

Pilkington K., et al., 2012a, MNRAS, 425, 969

Pilkington K., et al., 2012b, A\&A, 540, A56

Planck Collaboration et al., 2014, A\&A, 571, A1

Rosa D. A., Dors O. L., Krabbe A. C., Hägele G. F., Cardaci M. V., Pastoriza M. G., Rodrigues I., Winge C., 2014, MNRAS, 444, 2005

Rosales-Ortega F. F., Díaz A. I., Kennicutt R. C., Sánchez S. F., 2011, MNRAS, 415, 2439

Rosito M. S., Tissera P. B., Pedrosa S. E., Rosas-Guevara Y., 2018, arXiv e-prints, p. arXiv:1811.11062

Rousseeuw P. J., Van Driessen K., 2006, Data Mining and Knowledge Discovery, 12, 29

Rupke D. S. N., Kewley L. J., Chien L. H., 2010, ApJ, 723, 1255

Sánchez-Menguiano L., et al., 2016, ApJ, 830, L40

Sánchez-Menguiano L., et al., 2017, A\&A, 603, A113

Schaller M., Dalla Vecchia C., Schaye J., Bower R. G., Theuns T., Crain R. A., Furlong M., McCarthy I. G., 2015, MNRAS, 454,2277

Schaye J., et al., 2015, MNRAS, 446, 521

Searle L., 1971, ApJ, 168, 327

Sillero E., Tissera P. B., Lambas D. G., Michel-Dansac L., 2017, MNRAS, 472, 4404

Spitoni E., Cescutti G., Minchev I., Matteucci F., Silva Aguirre V., Martig M., Bono G., Chiappini C., 2018, arXiv e-prints, p. $\operatorname{arXiv:1811.11196}$

Springel V., 2005, MNRAS, 364, 1105

Tissera P. B., White S. D. M., Scannapieco C., 2012, MNRAS, 420,255 


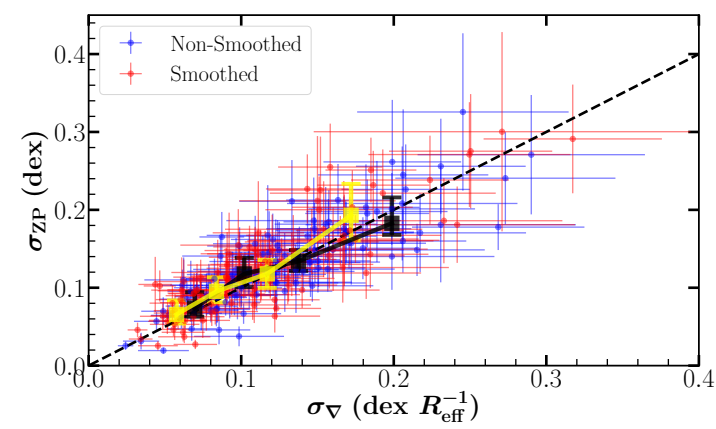

Figure A1. Median values of the standard deviations of the azimuthal zero points, $\sigma_{\mathrm{ZP}}$, as a function of those of the slopes, $\sigma_{\nabla}$, for the non-smoothed (black squares) and smoothed (yellow squares) metallicity profiles. The black (yellow) error bars represent the 25 and 75 percentile of the non-smoothed (smoothed) relations. The blue (red) small dots and blue (red) error bars represent the data for individual simulated galaxies and the corresponding errors obtained by applying a bootstrap sampling technique over the six azimuthal subregions.

Tissera P. B., Pedrosa S. E., Sillero E., Vilchez J. M., 2016, MNRAS, 456, 2982

Tissera P. B., Rosas-Guevara Y., Bower R. G., Crain R. A., del P Lagos C., Schaller M., Schaye J., Theuns T., 2019, MNRAS, 482,2208

Trayford J. W., Schaye J., 2019, MNRAS, 485, 5715

Trayford J. W., Frenk C. S., Theuns T., Schaye J., Correa C., 2019, MNRAS, 483, 744

Vogt F. P. A., Pérez E., Dopita M. A., Verdes-Montenegro L., Borthakur S., 2017, A\&A, 601, A61

Wiersma R. P. C., Schaye J., Smith B. D., 2009a, MNRAS, 393, 99

Wiersma R. P. C., Schaye J., Theuns T., Dalla Vecchia C., Tornatore L., 2009b, MNRAS, 399, 574

Zaritsky D., Kennicutt Robert C. J., Huchra J. P., 1994, ApJ, 420,87

Zinchenko I. A., Pilyugin L. S., Grebel E. K., Sánchez S. F., Vílchez J. M., 2016, MNRAS, 462, 2715

\section{APPENDIX A: ANALYSIS OF THE METALLICITY DISPERSION}

The EAGLE Project provides smoothed variables of the chemical abundances estimated by using the kernel function adopted for the Smoothed Particle Hydrodynamics calculations. These smoothed variables are estimated at the time the stars are formed. They provide a rough estimation of the effects that a more efficient mixing process might have. We re-do all the calculations to assess if the trend changed when the smoothed abundances are used instead of the nonsmoothed variables. However, no significant or systematic differences are found. As an example, in Fig. A1 we show the median dispersion in the slope and zero point of the metallicity gradients for both cases. Similarly, in Fig. A2 we show the slope and zero points obtained by using the star formation regions with excess or deficit metallicity with respect to the corresponding averages. As can be seen, there are no statistical differences between the results obtained from both relations.


Figure A2. Upper panel: $\nabla_{\delta_{+}}$and $\nabla_{\delta_{-}}$as a function of $\nabla_{\mathrm{T}}$ estimated for the whole distribution of super-young stars using the non-smoothed and smoothed chemical abundances. The black dashed line represents the 1:1 relation. The dashed lines depict linear regressions to the distributions of $\nabla_{\delta_{+}}$and $\nabla_{\delta_{-}}$for the smoothed and non-smoothed relations. Lower panel: $Z P_{+}$and $Z P_{-}$ as a function of $Z P_{\mathrm{T}}$. The black dashed lines represent a $1: 1$ relation, for comparison purposes. 